\title{
Second Order Advantage Applied to the Spectrophotometric Analysis of Ciprofloxacin and Dexamethasone in Ophthalmic Drops; Automatic Green Method Using On-line Photodegradation
}

\author{
Mariela F. Razuc, Marcos GrünhUt, Mariano GaRRIdo, and Beatriz S. Fernández Band ${ }^{\dagger}$ \\ Laboratory of Flow Injection Analysis, INQUISUR (Universidad Nacional del Sur-CONICET) \\ Av. Alem 1253 (B8000CPB), Bahía Blanca, Argentina
}

\begin{abstract}
On-line photodegradation and spectrophotometric analysis assisted by multivariate curve resolution-alternating least squares (MCR-ALS) was developed the simultaneous determination of ciprofloxacin (CIP) and dexamethasone (DEX) in ophthalmic suspensions using an automated flow-batch analysis (FBA) system. CIP and DEX have strongly overlapped UV spectra. Overcoming this lack of selectivity involves augmenting data dimensionality. This could be performed by adding information about the sample photodegradation to obtain the so-called second order advantage. Commercial sample analysis was successfully performed and no statistical differences $(\alpha=0.05)$ with respect to pharmacopeia methods were obtained. The proposed method offers several advantages over the methods developed to date. In agreement with the principles of green chemistry, only water was used as solvent, low amounts of waste were generated and on-line waste treatment was included in the system. Moreover, the cost per analysis was significantly reduced compared to methods that employ separative techniques.
\end{abstract}

Keywords Photodegradation, second order advantage, flow-batch, green chemistry, ciprofloxacin, dexamethasone

(Received August 8, 2014; Accepted October 13, 2014; Published December 10, 2014)

\section{Introduction}

Ciprofloxacin (CIP, 1-cyclopropyl-6-fluoro-1,4-dihydro-4-oxo7-(piperazin-1-yl)-3-quinoline carboxylic acid) $)^{1,2}$ and dexamethasone (DEX, 9-fluoro-11b,17,21-trihydroxy-16 $\alpha$ methylpregna-1,4-diene-3,20-dione) are employed together in several antiinfective eye drops to treat a wide variety of ophthalmic infection diseases like acute and sub acute conjunctivitis, keratitis and corneal ulcers caused by susceptible strains. ${ }^{1-3}$

Usually, CIP and DEX are quantified using high performance liquid chromatography (HPLC), 3,4 which is normally time consuming and uses relatively high amounts of organic solvents. For this reason, it is important to develop alternative methods able to quantify these analytes in a simple, economic, fast and environmental friendly way. ${ }^{5}$

Particularly, flow-batch analysis (FBA ${ }^{6}$ systems constitute an excellent alternative to automate methods used for the quality control of pharmaceuticals. FBA shows good precision and accuracy, high sample throughput, and low contamination, consumption, manipulation of reagents and samples, cost per analysis, and waste liberation for the environment. ${ }^{7,8}$ These aspects are in accordance with the concept of green analytical chemistry. ${ }^{9}$

On the other hand, most of the drugs in pharmaceuticals present absorption in the UV region. However, the lack of

† To whom correspondence should be addressed.

E-mail: usband@criba.edu.ar selectivity of the UV absorption bands could also be overcome by extending the dimensionality of the UV measurements when chemometric techniques are used. In this sense, the application of photochemical reactions can be used to improve analyte identification and quantitation in multicomponent mixtures. ${ }^{10}$ CIP and DEX undergo photodegradation in aqueous solutions after irradiation with a high-pressure mercury lamp or after exposure to day light. ${ }^{11,12}$

In this paper, we propose an automated, environmental friendly and low cost method for the simultaneous determination of CIP and DEX in ophthalmic suspensions. On-line photodegradation was performed in an FBA system coupled to a diode array UV detector. The UV spectra recorded throughout the time were arranged in matrices and subsequently analyzed using multivariate curve resolution optimized by alternating least squares (MCR-ALS) ${ }^{13}$ exploiting the so-called second order advantage. ${ }^{14}$ This advantage refers to the capacity of certain second-order algorithms to predict concentrations of sample components in the presence of any number of interferences. The lack of selectivity typical of the UV measurements was improved by including, in the data set, information related to the photodegradation reaction of CIP and DEX. The results obtained were compared with those obtained by pharmacopeia methods. ${ }^{15,16}$ Moreover, the proposed method was compared with respect to pharmacopeial and alternative methods $^{3-5}$ in terms of cost and environmental impact. Among these alternative methods, we considered two chromatographic determinations and also a method proposed in our previous article. $^{5}$ This method differs from the currently proposed method in the design of the FBA system, which did not include 


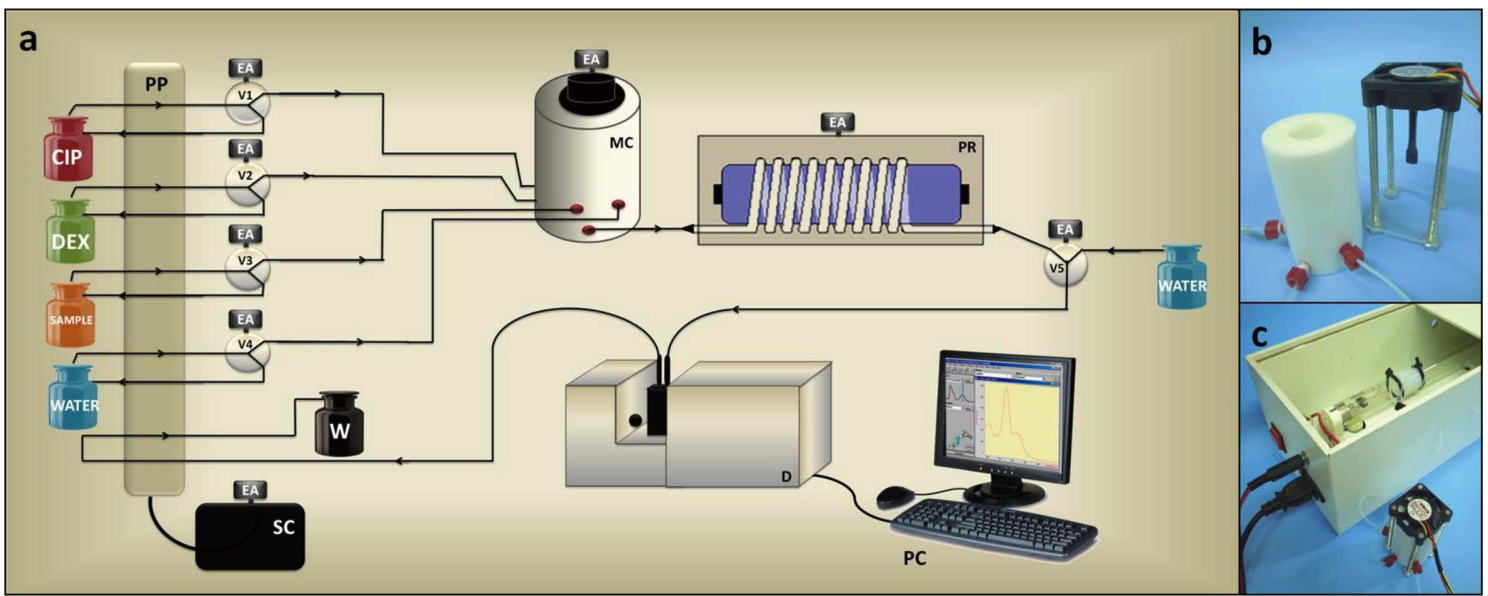

Fig. 1 a. Diagram of the FBA system, CIP. Ciprofloxacin solution, D. Detector, DEX. Dexamethasone solution, EA. Electronic actuator, MC. Mixing chamber, PC. Microcomputer, PP. Peristaltic pump, PR. Photoreactor, SC. Speed controller, V. Solenoid valve, W. Waste, The arrows indicate the direction of the fluids. b. Lab-made mixing chamber (MC) and stirring system, c. Lab-made photoreactor (PR).

a photodegradation step and used a mixing/detection chamber. Moreover, the kind of data obtained was also different. Instead of second order data, in our previous article, first order data were obtained and these were analyzed with the aid of chemometric tools, such as partial least squares and multiple linear regression.

\section{Experimental}

\section{Apparatus and software}

Spectrophotometric measurements were performed using an Agilent $^{\circledR} 8453$ UV-Vis diode array spectrophotometer. Fluids were pumped with a Gilson ${ }^{\circledR}$ Minipuls 3 peristaltic pump. A Hellma $^{\circledR}$ 178-712-QS flow cell was used. NResearch ${ }^{\circledR}$ threeway solenoid valves were used as selection valves. A lab-made mixing chamber (MC) coupled to a lab-made stirrer system was used. A lab-made photoreactor with a Phillips ${ }^{\circledR}$ low mercury UV lamp (15 W, $254 \mathrm{~nm})$ was used. An electronic actuator (EA) was used to control the peristaltic pump, valves, stirrer system and the photoreactor.

The software used for controlling the FBA system was developed in Labview ${ }^{\circledR}$ 5.1. Data treatment was performed using MATLAB ${ }^{\circledR} 7.0$ and the MCR-ALS subroutines. ${ }^{17}$

HPLC procedures were performed on a Gilson ${ }^{\circledR}$ liquid chromatograph equipped with a diode array UV 170 Gilson $^{\circledR}$ detector. A $5 \mathrm{~m} \mathrm{Restek}{ }^{\circledR} \mathrm{C} 18(250 \times 4.6 \mathrm{~mm}$ i.d. $)$ and a $5 \mathrm{~m}$ $\mathrm{Gemini}^{\circledR} \mathrm{C} 18(250 \times 4.6 \mathrm{~mm}$ i.d. $)$ analytical columns were used for CIP and DEX analyses, respectively.

\section{Reagents and solutions}

Analytical reagent-grade chemicals and ultra pure deionized water $(18.3 \Omega \mathrm{cm}$, Barnstead, Dubuque, IA) were used. Stock solutions of $24.0 \mathrm{mg} \mathrm{L}^{-1}$ ciprofloxacin hydrochloride (Saporiti, Buenos Aires, Argentina) and $8.0 \mathrm{mg} \mathrm{L}^{-1}$ dexamethasone (Saporiti, Buenos Aires, Argentina) were prepared in water. Stock solutions were protected from light and stored at $4{ }^{\circ} \mathrm{C}$. Working standard solutions were prepared daily by appropriate dilutions of the stock solutions in water.

The commercial ophthalmic drops analyzed were Fotamicin ${ }^{\circledR}$ (Elea), Decadron con Ciprofloxacina ${ }^{\circledR}$ (Sidus), and Procalm ${ }^{\circledR}$ (Atlas). The nominal concentrations, in all cases, were
$3.0 \mathrm{mg} \mathrm{mL}^{-1}$ for CIP and $1.0 \mathrm{mg} \mathrm{mL}^{-1}$ for DEX.

Preparation of the calibration set, validation set and samples A calibration set containing five mixtures of CIP and DEX was prepared in accordance with a full factorial design. The concentrations ranged between 3.2 and $8.0 \mathrm{mg} \mathrm{L}^{-1}$ for CIP and between 1.1 and $2.7 \mathrm{mg} \mathrm{L}^{-1}$ for DEX. Solutions were prepared taking into account the usual CIP-DEX relationship in the commercial pharmaceutical products. In order to evaluate the error of prediction, a validation set containing four mixtures was prepared in concentrations included within those of the calibration set.

Samples were prepared by diluting $200 \mu \mathrm{L}$ of the ophthalmic suspension, previously homogenized, with water up to $25 \mathrm{~mL}$. All samples were prepared in triplicate.

\section{FBA system}

The FBA system consisted of five channels (Fig. 1a). In four of them, CIP, DEX, the sample and water flowed toward the MC. In the fifth channel, the solutions flowed from the MC toward the photoreactor (PR) and the detector (D), and finally to the waste $(\mathrm{W})$. The direction of the flow was controlled by a three-way solenoid valve. Thus, for CIP, DEX, the sample and water, when the corresponding valve (i.e. $\mathrm{V}_{1}, \mathrm{~V}_{2}, \mathrm{~V}_{3}$ and $\mathrm{V}_{4}$, respectively) was OFF, the solution was recycled to the respective flask. When the valves were $\mathrm{ON}$, the solutions were pumped to the MC. The fifth valve $\left(\mathrm{V}_{5}\right)$ was used to control the emptying of the MC.

The MC was designed with four inlets and one outlet and its inner volume was $3.0 \mathrm{~mL}$. A stirring system was assembled on the top of the MC in order to obtain a rapid and efficient mixture of the solutions (Fig. 1b). It was made coupling a Teflon ${ }^{\circledR}$ stirrer blade to a cooler motor obtained from an Intel ${ }^{\circledR}$ microprocessor (DC $12 \mathrm{~V}, 0.06 \mathrm{~A}$ ).

In addition, a lab-made photoreactor was designed (Fig. 1c). In this device, $4.5 \mathrm{~m}$ of a Teflon ${ }^{\circledR}$ tube was helically arranged around the UV lamp. The switching of the photoreactor was controlled by software.

The developed software made it possible to program different sequences and operation times because of its flexible design. 
Table 1 Switching time intervals for the FBA system (expressed in $\mathrm{s}$ )

\begin{tabular}{lccccccr}
\hline & $\mathrm{V}_{1}$ & $\mathrm{~V}_{2}$ & $\mathrm{~V}_{3}$ & $\mathrm{~V}_{4}$ & Stirring & $\mathrm{V}_{5}$ & $\begin{array}{c}\mathrm{UV} \\
\text { lamp }\end{array}$ \\
\hline Blank & 0 & 0 & 0 & 60 & 60 & 200 & 0 \\
Calibration & $9-21$ & $9-21$ & 0 & $20-44$ & 60 & 200 & 360 \\
Validation & $12-18$ & $12-18$ & 0 & $26-38$ & 60 & 200 & 360 \\
Samples & 0 & 0 & 15 & 46 & 60 & 200 & 360 \\
\hline
\end{tabular}

\section{FBA procedure}

Firstly, the valves were set in a way that all solutions in their respective channels were pumped toward their flasks (OFF position). Then, $\mathrm{V}_{1}, \mathrm{~V}_{2}, \mathrm{~V}_{3}$ and $\mathrm{V}_{4}$ valves were switched $\mathrm{ON}$ for $5 \mathrm{~s}$ and the solutions were pumped towards the MC in order to fill the channels between the valves and the MC.

In order to obtain the blank signal, the valve $\mathrm{V}_{4}$ was switched $\mathrm{ON}$ for $60 \mathrm{~s}$. After that, water was pumped from the MC towards the $\mathrm{D}$ and the blank was measured.

Preparation of calibration and validation mixtures were performed by sequentially switching $\mathrm{ON}$ the $\mathrm{V}_{1}, \mathrm{~V}_{2}$ and $\mathrm{V}_{4}$ valves during previously defined intervals of time (Table 1). Simultaneously, the mixture was homogenized by stirring. After that, the $\mathrm{V}_{5}$ valve was switched $\mathrm{ON}$ for $200 \mathrm{~s}$ in order to fill the photoreactor with the mixture coming from the MC. When the photoreactor was filled, the UV lamp was switched ON. Immediately, spectra between 200 and $353 \mathrm{~nm}$ were registered every $2 \mathrm{~s}$ for a duration of $360 \mathrm{~s}$. After each measurement, the mixture was aspirated toward the waste. The same procedure was performed to prepare the samples. Then, the $\mathrm{V}_{3}$ and $\mathrm{V}_{4}$ valves were switched $\mathrm{ON}$ during a corresponding interval of time (Table 1). In all cases, the total volume added into the MC was the same, i.e. $1.0 \mathrm{~mL}$.

The system was always cleaned between measurements. The $\mathrm{MC}$ cleaning procedure was performed by switching $\mathrm{ON}$ the $\mathrm{V}_{4}$ valve and stirring for $60 \mathrm{~s}$. The total emptying of the MC was assured by switching $\mathrm{ON}$ the $\mathrm{V}_{5}$ valve for $200 \mathrm{~s}$ (Table 1).

\section{Data arrangement and pretreatment}

The photodegradation was performed on the calibration set and over the commercial samples (only in one replicate of each commercial brand). The data obtained through each photodegradation reaction were arranged in individual matrices $(181 \times 153)$. The validation set and two replicates of each commercial sample were not photodegraded; in such cases a unique spectrum was recorded.

The individual matrices and the spectra from the non-degraded solutions were arranged in a column-wise augmented data matrix D $(1466 \times 153)$.

Spectral data were smoothed using the Savitsky-Golay algorithm ${ }^{18}$ with a second order polynomial and an overall window size of 7 points.

The number of factors $(\mathrm{N})$ was estimated by singular value decomposition (SVD). ${ }^{19}$ The chemical rank was estimated by simply inspecting the singular values plot for the augmented matrices.

Initial estimates were obtained by applying the simple-to-use interactive self-modeling mixture analysis (SIMPLISMA) algorithm, a technique based on selecting the purest variables. ${ }^{20}$

For the MCR-ALS algorithm, ${ }^{21-24}$ the constraints were: a) nonnegativity constraint for spectra, b) non-negativity constraint for the concentration profiles, c) unimodality constraint for concentration profiles, d) equality constraints for spectra.
A linear model for CIP and DEX was obtained by making a regression of the area under each profile of the calibration set in the concentration matrix recovered by MCR-ALS (matrix $\mathbf{C}$ ) against the known concentration values. The areas under the profiles obtained for the validation set in matrix $\mathbf{C}$ were interpolated in the straight line obtained in the calibration step in order to obtain the prediction error. The areas of the sample set made it possible to obtain the unknown concentration of the analytes in the commercial samples.

The parameters used to indicate the quality of the models were the percentage of lack of fit (\% LOF) and the percentage of explained variance (EV), which are defined elsewhere. ${ }^{25}$ Another parameter calculated was the root mean square error (RMSE):

$$
\operatorname{RMSE}=\left[\frac{\sum_{i=1}^{l}\left(C_{\mathrm{nom}}-C_{\mathrm{pred}}\right)^{2}}{l}\right]^{1 / 2}
$$

where $C_{\text {nom }}$ and $C_{\text {pred }}$ represent the nominal and predicted concentrations, respectively, and $I$ is the total number of standards of calibration (or validation).

\section{Chromatographic procedure}

The chromatographic procedure was performed for CIP and DEX according to the established reference methods. ${ }^{15,16}$ The CIP determination was performed in isocratic mode. A mixture of phosphoric acid $\left(0.025 \mathrm{~mol} \mathrm{~L}^{-1}, \mathrm{pH}=3\right)$ and acetonitrile (87:13) was used as the mobile phase. The column was thermostated at $40^{\circ} \mathrm{C}$ and the flow rate was $1.5 \mathrm{~mL} \mathrm{~min}^{-1}$. The sample volume injected was $20 \mu \mathrm{L}$ and the signals were recorded at $278 \mathrm{~nm}$.

For DEX determination, the chromatographic procedure was performed also in isocratic mode using a 60:40 mixture of water and acetonitrile as the mobile phase. The column was thermostated at $25^{\circ} \mathrm{C}$ and the flow rate was $2.0 \mathrm{~mL} \mathrm{~min}{ }^{-1}$. The sample volume injected was $20 \mu \mathrm{L}$ and the signals were recorded at $254 \mathrm{~nm}$.

The standard solutions were prepared by dissolving CIP and DEX in their respective mobile phases. The peaks of the analytes were well-resolved and tailless. Retention times were $12.8 \mathrm{~min}$ and $16.5 \mathrm{~min}$ for CIP and DEX, respectively. Determinations were performed in triplicate.

\section{Results and Discussion}

\section{Spectral features and photodegradation study}

CIP and DEX aqueous solutions showed strong spectral absorption between $210-370 \mathrm{~nm}$ and $210-300 \mathrm{~nm}$, respectively. Therefore, the mixture of both analytes showed serious overlapping, with the DEX spectrum totally overlapped by the CIP spectrum. ${ }^{5}$

It is known from previous articles that CIP is photodegraded in the presence of UV radiation. The photodegradation of CIP is $\mathrm{pH}$-dependent; the process is considerably faster at $\mathrm{pH} 7$, near the isoelectric point, and slower at acid and alkaline $\mathrm{pH}$ levels. ${ }^{12}$ The photodegradation of DEX was studied in the same conditions used for CIP degradation. Thus, solutions of $5.6 \mathrm{mg} \mathrm{L}^{-1}$ for CIP, $1.9 \mathrm{mg} \mathrm{L}^{-1}$ for DEX and a CIP-DEX solution in the same concentrations were prepared in aqueous medium in agreement with the CIP-DEX relationship found in the analyzed pharmaceutical products. Figures $2 \mathrm{a}, 2 \mathrm{~b}$ and $2 \mathrm{c}$ are 3D graphics of CIP, DEX and CIP-DEX photodegradation, 
a

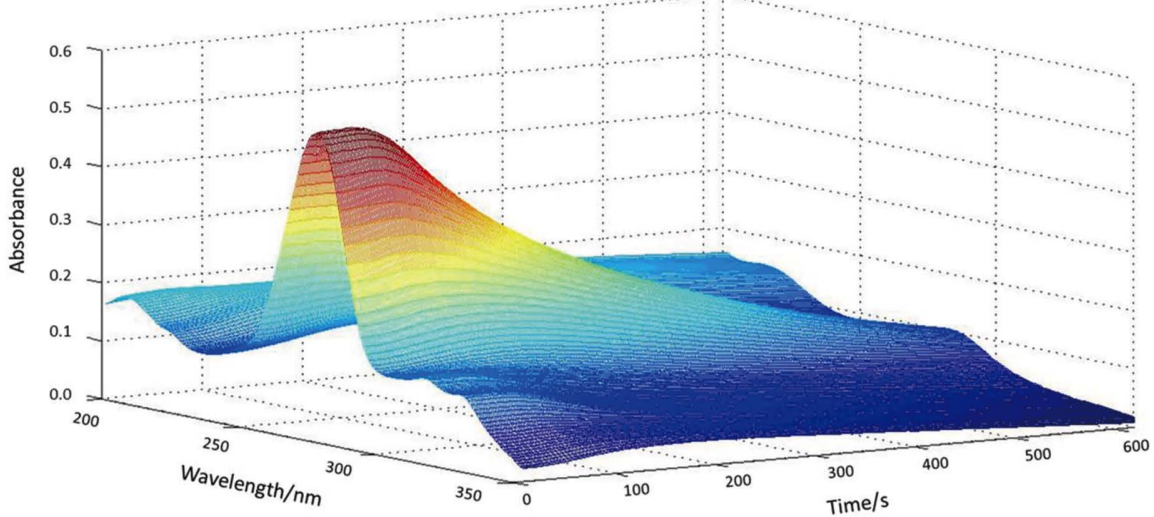

b

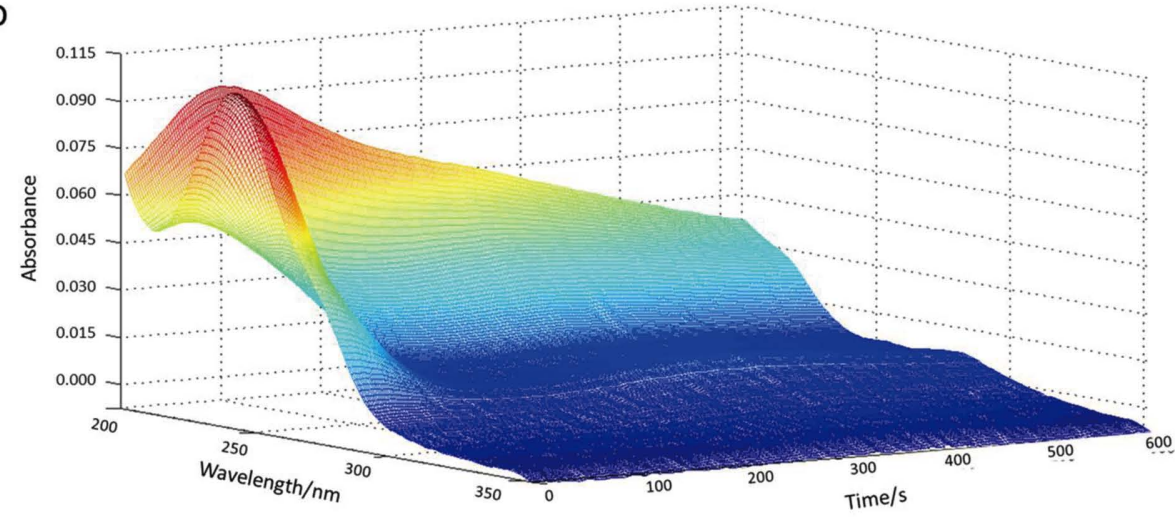

C

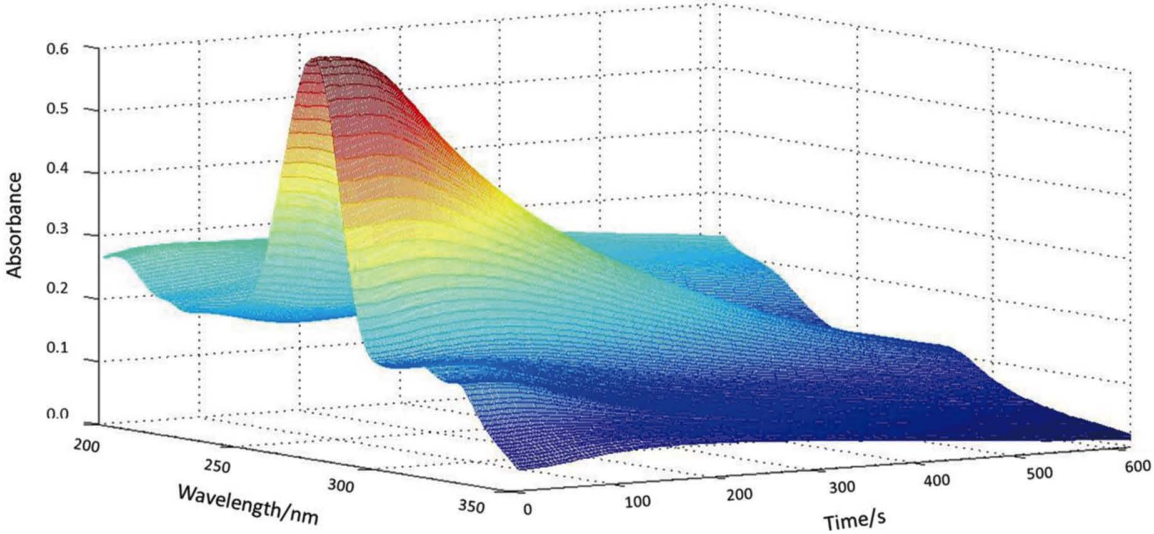

Fig. 2 Spectra of a) CIP/5.6 $\mathrm{mg} \mathrm{L}^{-1}$, b) DEX/1.9 $\mathrm{mg} \mathrm{L}^{-1}$ and c) CIP-DEX/5.6 - $1.9 \mathrm{mg} \mathrm{L}^{-1}$, solutions recorded throughout the photoreaction time.

respectively, as a function of photodegradation time. As can be seen, both analytes photodegraded under UV radiation, showing marked changes in their spectra in a short period. The CIP spectrum shows the more important changes between 260 and $300 \mathrm{~nm}$, where the signal decreases as a function of time. For DEX, the absorbance increases between 200 and $210 \mathrm{~nm}$ at the beginning of the photodegradation, and decreases after $100 \mathrm{~s}$ of reaction time. However, the more important changes in the DEX spectrum take place between 210 and $280 \mathrm{~nm}$, where the signal decreases quickly at the beginning of the photodegradation and remains almost unaltered after $100 \mathrm{~s}$.

\section{Optimization of FBA system}

The FBA system was used to perform the automatic preparation of the calibration and validation mixtures and samples, reducing the total time of analysis. For this purpose, the optimal volume in the MC should be the lowest possible. It was selected as a compromise between the reproducibility in the preparation of solutions and an appropriate time to empty the MC. Thus, the optimal volume selected was $1.0 \mathrm{~mL}$. Flow rates optimization was performed in order to obtain a short time of analysis and maximum precision in the preparation of standard solutions and samples. Thus, the flow rates for each channel were set at $0.94,0.92,0.94$ and $1.00 \mathrm{~mL} \mathrm{~min}^{-1}$ for CIP, DEX, the sample and water, respectively. On the other hand, the flow rate used to fill the photoreactor was $0.30 \mathrm{~mL} \mathrm{~min}^{-1}$ and the one used for the photodegradation process and detection was $0.08 \mathrm{~mL} \mathrm{~min}{ }^{-1}$. On the basis of the optimized variables, the calculated sample throughput was $6 \mathrm{~h}^{-1}$. 


\section{Data analysis}

Good resolution of the system depends strongly on the correct selection of $\mathrm{N}$. In the absence of other sources of variability (e.g. instrumental drift or noise), it could be assumed that $\mathrm{N}$ is equal to the number of absorbing species present in the system. In order to obtain the $\mathbf{N}$ value, SVD was applied to the $\mathbf{D}$ matrix and it suggested that there are three significant factors. Two of these components could be assigned to the presence of CIP and DEX. The third component observed would correspond to a photoproduct generated during the photodegradation. Afterwards, for the ALS optimization we started using the spectra provided by the SIMPLISMA algorithm as initial estimates. Information about the presence/absence of the analytes in the calibration, validation and samples solutions was given. It was assumed that the third component of $\mathbf{D}$ was present in all the photodegradation matrices.

Three spectral profiles were obtained after the application of MCR-ALS to D matrix (Fig. 3). CIP and DEX spectra can be clearly observed (dotted and solid lines, respectively). These spectra are equal to the CIP and DEX spectra at $t=0 \mathrm{~s}$ of photodegradation in Figs. $2 a$ and $2 b$, respectively. The third spectrum profile has a similar shape to the spectrum recovered

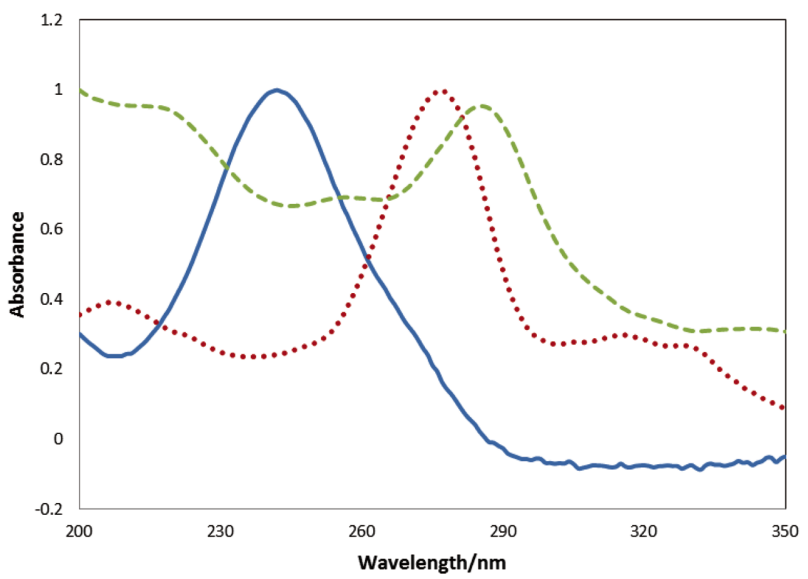

Fig. 3 Spectra recovered when augmented matrix $\mathbf{D}$ was analyzed by MCR-ALS. Dotted line: CIP spectrum; solid line: DEX spectrum; dashed line: spectrum associated to a CIP photoproduct. for a CIP photoproduct arising from the reductive dehalogenation of CIP. ${ }^{12}$ The similarity coefficient between the spectrum corresponding to this photoproduct and the one recovered by MCR-ALS for the third component in D matrix was 0.9999, corroborating this assumption.

Furthermore, Fig. 4 shows the concentration profiles obtained after the application of MCR-ALS to D matrix. The first fifth plots correspond to the concentration profiles of the calibration set (Figs. $4 \mathrm{a}-4 \mathrm{e}$ ), and the following ones (Figs. $4 \mathrm{f}-4 \mathrm{~h}$ ) are the concentration profiles recovered for the samples. In all cases, CIP and DEX concentrations decrease as a function of the photodegradation time, whereas the photoproduct concentration profiles increase over time.

Table 2 shows the quality parameters for the models obtained when MCR-ALS was applied to $\mathbf{D}$ matrix. The EV and LOF

Table 2 Results obtained through the MCR-ALS analysis of $\mathbf{D}$ matrix

\begin{tabular}{lc}
\hline & MCR-ALS \\
\hline Spectral region/nm & $200-352$ \\
$\mathrm{~N}$ & 3 \\
Model evaluation & 3.9 \\
LOF/\% & 99.85 \\
$E V / \%$ & \\
Calibration & \\
CIP & $3.20-8.00$ \\
Concentration range/mg L & \\
Linear equation & $0.9130 * x+0.0845$ \\
RMSEC $/ \mathrm{mg} \mathrm{L}^{-1}$ & 0.0903 \\
DEX & $1.10-2.70$ \\
Concentration range/mg L & \\
Linear equation & $0.0134 * x+0.0084$ \\
RMSEC $/ \mathrm{mg} \mathrm{L}^{-1}$ & 0.0367 \\
Validation & \\
CIP & \\
Concentration range/mg L & \\
RMSEP $/ \mathrm{mg} \mathrm{L}^{-1}$ & $4.40-6.80$ \\
DEX & 0.0922 \\
Concentration range/mg L & \\
RMSEPb/mg L & \\
\hline
\end{tabular}

a. RMSEC: root mean square error of calibration, b. RMSEP: root mean square error of prediction.

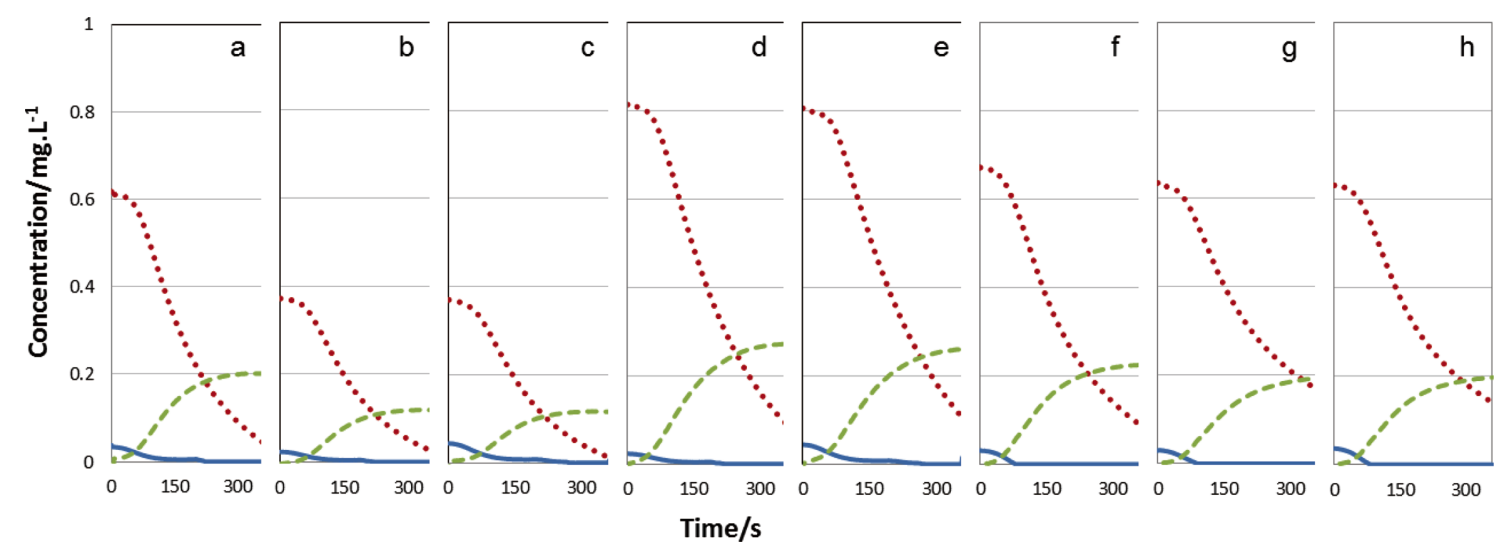

Fig. 4 Kinetic profiles recovered when augmented matrix $\mathbf{D}$ was analyzed by MCR-ALS. Dotted line: CIP spectrum; solid line: DEX spectrum; dashed line: spectrum associated to a CIP photoproduct. $\mathrm{a}$ - e: concentration profiles of the calibration set; $\mathrm{f}$ - $\mathrm{h}$ : concentration profiles recovered for the samples. 
(\%) values obtained for the model were satisfactory. The parameters related to the calibration performance and prediction ability were acceptable for both CIP and DEX. In order to investigate for the possible presence of overfitting, the MCRALS models were performed taking into account one component less and one component more than the suggested one by SVD. When two components were considered, the values of LOF (\%) and $\mathrm{EV}(\%)$ obtained were slightly worse (i.e. 5.1 and $99.73 \%$, respectively), and the parameters of prediction were worse as well. On the other hand, for four components, non-convergence was obtained in the algorithm. Therefore, the obtained model should be able to quantify CIP and DEX equally well.

\section{Application to real samples}

The proposed method was successfully applied to the simultaneous determination of CIP and DEX in ophthalmic drops. The RSD\% $(n=3)$ obtained from the sample analysis was 3.5 and 7.9 for CIP and DEX, respectively. Table 3 shows the obtained results for both analytes by the proposed method and pharmacopeial methods..$^{15,16}$ The statistical comparison was performed using a paired $t$-test. Non-significant differences

Table 3 Analysis of CIP and DEX in ophthalmic drops

\begin{tabular}{|c|c|c|c|c|c|c|}
\hline & \multicolumn{2}{|c|}{ Nominal } & \multicolumn{2}{|c|}{ HPLC $^{15,16}$} & \multicolumn{2}{|c|}{$\begin{array}{c}\text { Proposed } \\
\text { method }\end{array}$} \\
\hline & CIP & DEX & CIP & DEX & CIP & DEX \\
\hline Fotamicin ${ }^{\circledR}$ & 300 & 100 & $295(2)$ & $94(1)$ & $304(2)$ & $91(2)$ \\
\hline Decadron con cipro $^{\circledR}$ & 300 & 100 & $309(2)$ & $96(2)$ & $310(1)$ & $99(2)$ \\
\hline Procalm $^{\circledR}$ & 300 & 100 & $329(2)$ & $99(2)$ & $326(2)$ & $107(1)$ \\
\hline
\end{tabular}

The concentrations are expressed in $\mathrm{mg} / 100 \mathrm{~mL}$. The samples were analyzed in triplicate. Standard deviations are in parenthesis. were obtained at a confidence level of $95 \%$.

\section{Green approach}

Green chemistry is related to the design of chemical products and processes that reduce or eliminate the use and generation of hazardous substances. ${ }^{26}$ Table 4 compares the proposed method with respect to pharmacopeial and some alternative methods developed to date to quantify CIP and DEX in ophthalmic suspensions. The current method meets several of the 12 principles of green chemistry. ${ }^{26}$ In this sense, in accordance with the $1^{\circ}, 5^{\circ}$ and $12^{\circ}$ principles, no reagent was used, the only solvent employed was water and low amounts of waste were generated in comparison with others methods (Table 4).

Moreover, the proposed method is the only one that presents on-line waste treatment and was performed by the on-line photodegradation of the solutions. The photodegradation significantly decreases the activity of $\mathrm{CIP}^{27}$ thus reducing the environmental impact caused by the antibiotic.

In terms of costs, the use of water as the solvent contributed significantly to reduce the costs of analysis. As can be seen in Table 4, the proposed method is much less expensive than the methods that require separative techniques.

\section{Conclusions}

Quantitative analysis of CIP and DEX in ophthalmic suspensions was successfully performed by an automated FBA system with an on-line photodegradation step and UV spectrophotometric detection assisted by MCR-ALS. The photodegradation of CIP and DEX allowed the data dimensionality to be extended, and the MCR-ALS analysis of such second order data made it possible to overcome the lack of selectivity typical of the UV absorption bands. Thus, both analytes were quantified using only one MCR-ALS model.

Table 4 Comparison of methods developed to quantify ciprofloxacina and dexamethasone

\begin{tabular}{|c|c|c|c|c|c|c|}
\hline \multirow[b]{2}{*}{ Analyte } & \multicolumn{2}{|c|}{ Pharmacopeial met hod ${ }^{15,16}$} & \multirow{2}{*}{$\frac{\text { Method } 1^{3}}{\text { CIP-DEX }}$} & \multirow{2}{*}{$\frac{\text { Method } 2^{4}}{\text { CIP-DEX }}$} & \multirow{2}{*}{$\frac{\text { Method } 3^{5}}{\text { CIP-DEX }}$} & \multirow{2}{*}{$\frac{\text { Proposed method }}{\text { CIP-DEX }}$} \\
\hline & CIP & DEX & & & & \\
\hline $\begin{array}{l}\text { Separative } \\
\text { technique }\end{array}$ & HPLC & HPLC & HPLC & HPLC & No & No \\
\hline Detection & $\begin{array}{c}\text { Spectrophotometric } \\
278 \mathrm{~nm}\end{array}$ & $\begin{array}{c}\text { Spectrophotometric } \\
254 \mathrm{~nm}\end{array}$ & $\begin{array}{l}\text { Spectrophotometric } \\
254 \mathrm{~nm}\end{array}$ & $\begin{array}{c}\text { Spectrophotometric } \\
254 \mathrm{~nm}\end{array}$ & $\begin{array}{c}\text { Spectrophotometric } \\
210-400 \mathrm{~nm} \\
(\lambda=2)\end{array}$ & $\begin{array}{c}\text { Spectrophotometric } \\
200-353 \mathrm{~nm} \\
(\lambda=2)\end{array}$ \\
\hline Solvents & $\begin{array}{l}\text { Phosphoric acid and } \\
\text { triethylamine: } \\
\text { acetonitrile }(87: 13)\end{array}$ & $\begin{array}{c}\text { Water:acetonitrile } \\
(75: 25)\end{array}$ & $\begin{array}{l}\text { Phosphate buffer: } \\
\text { acetonitrile (65:35) }\end{array}$ & $\begin{array}{c}\text { Ethanol:water: } \\
\text { triethylamine } \\
(55: 45: 0.6)\end{array}$ & Water & Water \\
\hline $\begin{array}{l}\text { On-line waste } \\
\text { treatment }\end{array}$ & No & No & No & No & No & Photodegradation \\
\hline $\begin{array}{l}\text { Analyte amount }{ }^{\mathrm{a} /} \\
\mu \mathrm{g}\end{array}$ & 1.2 & 0.8 & $0.3-0.1$ & $6.0-2.0$ & $7.2-2.4$ & $5.6-1.9$ \\
\hline $\begin{array}{l}\text { Sample } \\
\text { throughput } / \mathrm{h}^{-1}\end{array}$ & 4 & 7.5 & 11 & 6 & 10 & 6 \\
\hline $\begin{array}{l}\text { Waste volume }{ }^{\mathrm{b}} / \\
\mathrm{mL}\end{array}$ & 22.5 & 15 & 5 & 8.8 & 3 & 1 \\
\hline Costc/USD & 5.21 & 3.98 & 1.89 & 8.23 & 0.36 & 0.51 \\
\hline
\end{tabular}

a. Injected amount per sample, b. Consumed volume of solvent per sample (no conditioning of the column was considered), c. Cost per sample. The values were calculated taking into account the cost per hour corresponding to the use of the instruments (according to tariff 2014, LIUC-Universidad Nacional del Sur) and the prices of the solvents and accessories employed for the analysis. 
The FBA system automatically performed the preparation of standards and samples and the photodegradation procedure, thus improving precision and reducing human intervention.

Ophthalmic drops were analyzed and the obtained results were in accordance with those obtained by the pharmacopeia method (HPLC), demonstrating the suitability of the proposed method.

In summary, this method offers several advantages over some of the methods developed to date, mainly in relation to automation, compliance with several of the principles of green chemistry, and the reduced cost per sample. For this reason, it represents an environmental friendly and economic alternative for the quality control of these pharmaceutical preparations.

\section{Acknowledgements}

The authors would like to acknowledge appreciation to Consejo Nacional de Investigaciones Cientificas y Técnicas (CONICET) and for financial support from the Universidad Nacional del Sur.

\section{References}

1. M. Le Bel, Pharmacotherapy, 1988, 8,3 .

2. E. Turiel, G. Bordin, and A. R. Rodríguez, J. Environ. Monit., 2005, 7, 189.

3. K. Prakash and K. R. Sireesha, J. Chem., 2012, 9, 1077.

4. U. H. Desai, A. H. Patwari, J. K. Maradiya, M. K. Sathawara, B. N. Suhagia, and I. S. Rathod, Int. J. Pharm. Sci. Drug Res., 2013, 5, 62.

5. M. F. Razuc, M. Grünhut, E. Saidman, M. Garrido, and B. S. Fernández Band, Talanta, 2013, 115, 314.

6. P. G. Dias Diniz, L. F. Almeida, D. P. Harding, and M. C. de Araújo, TrAC, Trends Anal. Chem., 2013, 35, 39.

7. B. F. Reis, M. F. Giné, E. A. G. Zagatto, J. L. F. C. Lima, and R. A. Lapa, Anal. Chim. Acta, 1994, 293, 129.

8. P. C. A. Jerônimo, A. N. Araujo, M. Montenegro, C. Pasquini, and I. M. Raimundo, Anal. Bioanal. Chem., 2004, 380, 108.

9. W. R. Melchert, B. F. Reis, and F. R. P. Rocha, Anal. Chim.
Acta, 2012, 714, 8.

10. Y. C. Kim, J. A. Jordan, M. L. Nahorniak, and K. S. Booksh, Anal. Chem., 2005, 77, 7679.

11. J. B. Belden, J. D. Maul, and M. J. Lydy, Chemosphere, 2007, 66, 1390.

12. M. F. Razuc, M. Garrido, Y. S. Caro, C. M. Teglia, H. C. Goicoechea, and B. S. Fernández Band, Spectrochim. Acta, Part A, 2013, 106, 146.

13. S. Steven, D. Brown, R. Tauler, and B. Walczak, "Comprehensive Chemometrics: Data preprocessing, linear soft-modeling, unsupervised data mining", 2009, Elsevier, Amsterdam.

14. K. S. Booksh and B. R. Kowalski, Anal. Chem., 1994, 66, 782A.

15. British Pharmacopeia, 2001, The Stationery Office Ltd., Norwich, 1951.

16. USP 34/ NF 29, 2011, United States Pharmacopeial Convention, Rockville, 2719.

17. Multivariate Curve Resolution Homepage, http://www. mcrals.info.

18. A. Savitzky and M. J. E. Golay, Anal. Chem., 1964, 36, 1627.

19. D. L. Massart, B. G. M. Vandeginste, L. M. C. Buydens, S. De Jong, P. J. Lewi, and J. Smeyers-Verbeke, "Handbook of Chemometrics and Qualimetrics: Part A", 1997, Elsevier, Amsterdam.

20. W. Windig and J. Guilment, Anal. Chem., 1991, 63, 1425.

21. R. Tauler, A. Izquierdo-Ridorsa, and E. Casassas, Chemom. Intell. Lab. Syst., 1993, 18, 293.

22. R. Tauler, A. Smilde, and R. Kowalsky, J. Chemom., 1995, 9, 31.

23. M. Grünhut, M. Garrido, M. E. Centurión, and B. S. Fernández Band, Anal. Chim. Acta, 2010, 673, 33.

24. V. A. Lozano and G. M. Escandar, Anal. Chim. Acta, 2013, 782, 37.

25. M. Garrido, I. Lázaro, M. S. Larrechi, and F. X. Rius, Anal. Chim. Acta, 2004, 515, 65.

26. P. T. Anastas and M. M. Kirchhoff, Acc. Chem. Res., 2002, 35, 686.

27. E. Fernández, G. Sánchez, E. Navarrete, and F. Del Alcázar, Ars. Pharm., 2004, 45, 111. 